feedback surveys. Reduced waste as meals are prepared as and when required.

Conclusions Working in end of life care you develop an appreciation early on that every meal prepared has to be the best. It could very well be the last meal a patient has. Evaluation of the room-service catering shows a positive contribution. We have also learnt how much our input can mean and matter to relatives and friends of patients. Seeing a loved one being cared for, not just through exceptional medical/nursing care, but through a dedicated catering team trying their hardest too, may just help to ease the pain they experience as well.

\section{P-110 LOVE ON A PLATE: A QUALITY IMPROVEMENT PROJECT EXPLORING NUTRITIONAL CARE}

Sharon Twigger, Suzanne Davies. Compton Care, Wolverhampton, UK

\subsection{6/bmjspcare-2018-hospiceabs. 135}

Background Good nutritional care is essential to the delivery of excellent palliative care. In addition to our bodies' physical needs, nutrition can have social, psychological and spiritual importance to patients. Despite this historically nutrition has not always been well supported in palliative care organisations (Help the Hospices Food and Nutrition Group, 2009).

Aim(s) To examine the current nutritional care provision within our organisation; to compare this to national standards; to explore where deficiencies existed and how these could be overcome.

Methods A full review of nutritional care was undertaken in 2017 across the organisation. Five key functional areas relating to nutrition were explored: catering management; patients' nutrition; communication; governance; and others' nutrition. Tools used included direct observation of catering processes, talking to key members of staff, seeking feedback from patients, completing the Hospice UK Nutrition and Hydration Audit Tool and visiting other local hospices delivering excellent nutritional care.

Results The review produced a number of recommendations for improvement across the five key functional areas. A Nutritional Care Steering Group was set up to ensure recommendations were delivered in a coordinated way and that strong governance structures underpinned this. Changes being implemented are already directly impacting positively on patient care.

Conclusions Delivery of excellent nutritional care requires a whole system approach where each aspect of the service is explored and improved cohesively.

\section{P-111 CAMBRIDGE BREATHLESSNESS INTERVENTION SERVICE: IMPACT ON HOSPITAL ATTENDANCE}

${ }^{1}$ Chloe Chin, ${ }^{2}$ Efthalia Massou, 'Julie Burkin, 'Sara Booth, ${ }^{1,2}$ Anna Spathis. ${ }^{1}$ Cambridge University Hospitals NHS Foundation Trust, Cambridge, UK; ${ }^{2}$ Department of Public Health and Primary Care, University of Cambridge, Cambridge, UK

\subsection{6/bmjspcare-2018-hospiceabs. 136}

Context Cambridge Breathlessness Intervention Service (CBIS) supports patients with intractable breathlessness due to a variety of conditions. This evidence-based, multidisciplinary, complex intervention promotes self-management of symptoms using predominantly non-pharmacological techniques. Successful self-management reduces Emergency Department attendance (EDA) and inpatient admissions. This service evaluation aimed to investigate the impact of CBIS on hospital attendances, in terms of EDAs and inpatient admissions.

Method The number of EDAs and the number and length of admissions in the six months before, and six months after, first clinical contact with CBIS were recorded for all patients referred during four specific months. Costs of $£ 138 / \mathrm{EDA}$ and $£ 306 /$ inpatient bed day were assumed. Other than in cost calculations, a 'bed day' was defined as either an EDA or an inpatient bed day. 'Avoidable attendances' were EDAs or admissions due to breathlessness alone without an underlying cause requiring intervention in hospital.

Results Data from 74 patients were analysed. There was a significant 43\% absolute risk reduction in hospital attendances $(p=0.001)$. There were 3.0 fewer bed days for each patient seen. Avoidable attendances also reduced significantly $(p=0.023)$. The odds of an avoidable attendance was three times lower after receiving CBIS (OR 0.31, p=0.049), and the odds of an avoidable bed day dropped significantly (OR 0.24 , $\mathrm{p}<0.001)$. The five fewer EDAs and 214 fewer inpatient bed days saves $£ 65484$ or $£ 894 /$ patient.

Discussion CBIS significantly reduced hospital attendance.

The intervention costs $£ 119 /$ patient, suggesting potential overall cost savings. Interestingly, all types of hospital attendances were reduced, not only those deemed potentially avoidable. CBIS supports patients to develop self-efficacy, and improves physical and psychological wellbeing. Therefore, the service may impact hospital attendance for a range of reasons, not only breathlessness. Further work is needed to gain insight into, and increase confidence, in these findings.

\section{P-112 INCREASING STAFF CONFIDENCE AND USE OF A HANDHELD FAN IN THE MANAGEMENT OF BREATHLESSNESS}

Margaret Kerr. Western Health and Social Care Trust (WHSCT), Derry/Omagh/Enniskillen, UK

10.1136/bmjspcare-2018-hospiceabs.137

Background Using a handheld fan has been identified in the literature as useful in the palliation of breathlessness (Luckett, Philips, Johnson et al., 2017).There is continuing evidence that the handheld fan should be recommended to all breathless patients as an evidenced based management strategy for their symptoms (Galbraith, Fagan, Perkins et al., 2010). It is also a useful intervention to recommend without qualification to any clinician or healthcare worker who sees breathless patients (Booth, Moffat \& Burkin, 2011). Yet we know within the WHSCT within palliative care settings where breathlessness is a symptom, it is not being commonly considered or used.

Aim To increase staff confidence in identifying, providing and teaching use of the fan. To increase the use of the handheld fan in the palliative care inpatient settings within the WHSCT (palliative care in-patient unit and hospice in-patient unit).

Methods Training package developed including educational video and practical teaching session and delivered to nursing staff. Staff confidence measured pre- and post- training, at three months and at one year post training. Numbers of fans provided monitored.

Results Training is currently on-going in both locations and will be completed by 30/06/2018 when pre- and post- training confidence scores will be collated. To date, initial data shows $100 \%$ of high confidence scores post training. It will be most 